\title{
Total synthesis of (+)-trans-195A
}

Nicole Holub, Jürgen Neidhöfer and Siegfried Blechert*

Technische Universität Berlin, Institut für Chemie

Strasse des 17. Juni 135

D-10623Berlin

Germany

\section{Supporting Information}

Experimental procedures and characterization data for compounds 1, 3-6, 8-12, 15, 18

(8 pages)

General experimental details. All reactions were carried out under nitrogen-atmosphere using flame dried glassware with the exception of CBS-reduction and Negishi-coupling. Metathesis reactions were performed in a Braun MB 150-G glove box under nitrogen. Commercial available reagents were used as received from Aldrich, Lancaster, Fluka and Acros Organics. THF was freshly distilled under $\mathrm{N}_{2}$ from sodium/benzophenone and DCM was distilled from $\mathrm{CaH}_{2}$. Analytical TLC was performed on Merck silica gel 60 (0.040-0.063 $\mathrm{mm}$ ) or Fluka alumina (type $\mathrm{H}$, neutral) and preparative chromatography was performed on ICN silica gel $60(0.040-0.063 \mathrm{~mm})$ or Fluka alumina (type 5016A basic, Brockmann activity grade 3). ${ }^{1} \mathrm{H}$ and ${ }^{13} \mathrm{C}$ NMR spectra were recorded on a Bruker AC 200 and DRX 500 spectrometer. Chemical shifts are reported to the internal solvent peak of $\mathrm{CDCl}_{3}$. HRMS were recorded on a Finnigan MAT 95 SQ spectrometer. IR spectra were measured on a Nicolet FTIR 750 spectrometer. Melting Points were determined on a Leica Galan III heater table microscope and are uncorrected. Elemental analyses were recorded on an Elementar Vario El Fa. HPLC analysis were taken on a Varian Pro Star 320 spectrometer with a chiral Daicel Chiracel-OJ column, $\lambda=254 \mathrm{~nm}$. Optical rotations were measured on a Perkin-Elmer polarimeter 341 at $25{ }^{\circ} \mathrm{C}$ and at a wavelength of $589 \mathrm{~nm}$.

(R)-1-Chloro-pentan-2-ol (8). To a stirred solution of (R)-epichlorohydrin 7 (99\%ee, $4.00 \mathrm{~g}$, $43.2 \mathrm{mmol})$ and $\mathrm{CuCN}(387 \mathrm{mg}, 4.32 \mathrm{mmol})$ in dry THF (50 mL), was added a $1 \mathrm{M}$ solution of ethylmagnesium bromide in THF $(21.6 \mathrm{~mL}, 64.9 \mathrm{mmol})$ dropwise at $-78{ }^{\circ} \mathrm{C}$. The mixture was warmed to $-20{ }^{\circ} \mathrm{C}$ over $3 \mathrm{~h}$ and poured into a saturated $\mathrm{NH}_{4} \mathrm{Cl}$ solution. The layers were separated and the aqueous layer was extracted with $\mathrm{Et}_{2} \mathrm{O}(3 \times 50 \mathrm{~mL})$. The combined ethereal extracts were dried over $\mathrm{MgSO}_{4}$ and concentrated to afford $\mathbf{8}$ as yellowish oil $(5.21 \mathrm{~g}, 98 \%)$ which was used without further purification; $[\alpha]_{D}^{20}-2.19\left(c 1.03, \mathrm{CHCl}_{3}\right) ; \mathbf{I R}(\mathrm{ATR}): v\left(\mathrm{~cm}^{-1}\right)$ 
3386, 2960, 2934, 2874, 1702; ${ }^{1} \mathbf{H}$ NMR (500 MHz): $\delta$ (ppm) 0.94 (t, $\left.J=7 \mathrm{~Hz}, 3 \mathrm{H}\right), 1.34$ $1.58(\mathrm{~m}, 4 \mathrm{H}), 2.11(\mathrm{bs}, 1 \mathrm{H}), 3.46(\mathrm{dd}, J=8,11 \mathrm{~Hz}, 1 \mathrm{H}), 3.63(\mathrm{dd}, J=3,11 \mathrm{~Hz}, 1 \mathrm{H}), 3.76-$ $3.87(\mathrm{~m}, 1 \mathrm{H}) ;{ }^{13} \mathrm{C}$ NMR $(125 \mathrm{MHz}): \delta(\mathrm{ppm}) 13.8\left(\mathrm{CH}_{3}\right), 18.6\left(\mathrm{CH}_{2}\right), 36.1\left(\mathrm{CH}_{2}\right), 50.4\left(\mathrm{CH}_{2}\right)$, 71.0 (CH); EI-MS (RT): m/z (\%) 123 (8), 121 (22), 105 (10), 86 (100); HRMS: calcd. for $\mathrm{C}_{5} \mathrm{H}_{10} \mathrm{ClO}[\mathrm{M}-\mathrm{H}]^{+}$121.0420, found 121.0421 .

(R)-2-Propyl-oxirane (9). To a solution of 8 (5.25 g, $42.8 \mathrm{mmol})$ in $^{\mathrm{Et}_{2} \mathrm{O}}$ (50 mL) was added finely powdered $\mathrm{NaOH}(9.42 \mathrm{~g}, 0.24 \mathrm{mmol})$. The mixture was stirred vigorously for $24 \mathrm{~h}$ and poured into $20 \mathrm{~mL}$ water. After separation of the layers, the aqueous layer was extracted with $\mathrm{Et}_{2} \mathrm{O}(3 \times 30 \mathrm{~mL})$ and the combined organic layers were dried over $\mathrm{MgSO}_{4}$. Removal of the solvent by distillation over a $10 \mathrm{~cm}$ vigreux-column afforded $\mathbf{9}(3.56 \mathrm{~g}, 96 \%)$ as yellowish oil, which was used without further purification; $[\alpha]_{D}^{20}+7.8\left(c 1.15, \mathrm{CHCl}_{3}\right)$; IR (ATR): $v\left(\mathrm{~cm}^{-1}\right)$ 3404, 2959, 2930, 2873, 1701, 1666, 1458; ${ }^{1} \mathbf{H}$ NMR (200 MHz): $\delta$ (ppm) 0.96 (t, $J=7 \mathrm{~Hz}$, $3 \mathrm{H}), 1.45-1.55(\mathrm{~m}, 4 \mathrm{H}), 2.44-2.47(\mathrm{~m}, 1 \mathrm{H}), 2.74(\mathrm{dd}, J=4,4 \mathrm{~Hz}, 1 \mathrm{H}), 2.86-2.95(\mathrm{~m}, 1 \mathrm{H}) ;{ }^{13} \mathbf{C}$ NMR (50 MHz): $\delta$ (ppm) $13.8\left(\mathrm{CH}_{3}\right), 19.1\left(\mathrm{CH}_{2}\right), 34.4\left(\mathrm{CH}_{2}\right), 46.9\left(\mathrm{CH}_{2}\right), 52.0(\mathrm{CH})$; EI-MS (RT): m/z (\%) 86 (19), 71 (9), 43 (100); HRMS: calcd. for $\mathrm{C}_{5} \mathrm{H}_{10} \mathrm{O}[\mathrm{M}]^{+}$86.0732, found 86.0731 .

(R)-Hept-1-en-4-ol (10). To a stirred solution of 9 (3.50 g, $40.6 \mathrm{mmol})$ and CuCN (364 mg, $4.06 \mathrm{mmol})$ in dry THF (30 mL), was added over $45 \mathrm{~min}$ a $1 \mathrm{M}$ solution of vinylmagnesium bromide in THF $(52.8 \mathrm{~mL}, 52.8 \mathrm{mmol})$ dropwise at $-78^{\circ} \mathrm{C}$. The mixture was allowed to warm up to $0{ }^{\circ} \mathrm{C}$, before it was quenched with a saturated $\mathrm{NH}_{4} \mathrm{Cl}$ solution $(20 \mathrm{~mL})$. The layers were separated, the aqueous layer extracted with $\mathrm{Et}_{2} \mathrm{O}(3 \times 50 \mathrm{~mL})$, the combined ethereal extracts were washed with brine $(20 \mathrm{~mL})$ and dried $\left(\mathrm{MgSO}_{4}\right)$. Evaporation of the solvent and chromatographic purification of the crude product (silica, $\mathrm{Et}_{2} \mathrm{O} /$ pentane $1: 3$ ) gave $\mathbf{1 0}$ as pale yellow oil $(4.41 \mathrm{~g}, 95 \%) ; \mathrm{R}_{\mathrm{f}}=0.46 ;[\alpha]_{D}^{20}+13.1$ (c 2.4, $\left.\mathrm{CHCl}_{3}\right)$; IR (ATR): $v\left(\mathrm{~cm}^{-1}\right) 3358$, 3077, 2959, 2931, 2873, 1641; ${ }^{1} \mathbf{H}$ NMR (500 MHz): $\delta(\mathrm{ppm}) 0.83(\mathrm{t}, J=7 \mathrm{~Hz}, 3 \mathrm{H}), 1.25-$ $1.42(\mathrm{~m}, 4 \mathrm{H}), 2.03-2.09(\mathrm{~m}, 1 \mathrm{H}), 2.15-2.20(\mathrm{~m}, 1 \mathrm{H}), 2.41$ (bs, 1H), 3.53-3.75 (m, 1H), 5.00 (d, $J=14 \mathrm{~Hz}, 1 \mathrm{H}), 5.03(\mathrm{~d}, J=14 \mathrm{~Hz}, 1 \mathrm{H}), 5.70-5.78(\mathrm{~m}, 1 \mathrm{H}) ;{ }^{13} \mathbf{C} \mathbf{N M R}(125 \mathrm{MHz}): \delta(\mathrm{ppm})$ $14.0\left(\mathrm{CH}_{3}\right), 18.8\left(\mathrm{CH}_{2}\right), 39.0\left(\mathrm{CH}_{2}\right), 42.1\left(\mathrm{CH}_{2}\right), 70.1(\mathrm{CH}), 117.7\left(\mathrm{CH}_{2}\right), 135.1(\mathrm{CH})$; EI-MS (RT): m/z (\%) 97 (2), 73 (96), 71 (31), 55 (100), 43 (84), 41 (59), 39 (34); HRMS: calcd. for $\mathrm{C}_{7} \mathrm{H}_{13}[\mathrm{M}-\mathrm{OH}]^{+}$97.1017, found 97.1022. 
(R)-Methanesulfonic acid 1-propyl-but-3-enyl ester (11). To an ice-cold stirred solution of $10(3.50 \mathrm{~g}, 30.7 \mathrm{mmol})$ and triethylamine $(4.69 \mathrm{~mL}, 33.7 \mathrm{mmol})$ in $\mathrm{CH}_{2} \mathrm{Cl}_{2}(35 \mathrm{~mL})$ was added dropwise methanesulfonyl chloride $(2.66 \mathrm{~mL}, 33.7 \mathrm{mmol})$ over $10 \mathrm{~min}$. The resulting mixture was allowed to warm up to r.t. and stirred for $3 \mathrm{~h}$. After diluting with $100 \mathrm{~mL}$ $\mathrm{CH}_{2} \mathrm{Cl}_{2}$, the solution was washed with water $(3 \mathrm{x} 50 \mathrm{~mL})$, brine $(40 \mathrm{~mL})$ and dried over $\mathrm{MgSO}_{4}$. Evaporation of the solvent and chromatographic purification (silica, MTBE/hexane 1:3) afforded $11(5.28 \mathrm{~g}, 90 \%)$ as pale yellow oil; $\mathrm{R}_{\mathrm{f}}=0.38 ;[\alpha]_{D}^{20}+27.4\left(c 1.0, \mathrm{CHCl}_{3}\right)$; IR (ATR): $v\left(\mathrm{~cm}^{-1}\right)$ 2962, 2939, 2876, 1643, 1331, 1172, 903; ${ }^{1}$ H NMR (200 MHz): $\delta$ (ppm) 0.93 $(\mathrm{t}, J=7 \mathrm{~Hz}, 3 \mathrm{H}), 1.31-1.49$ (m, 2H), 1.56-1.73 (m, 2H), 2.44-2.50 (m, 2H), 2.99 (s, 3H), 4.73 (quin, $J=6 \mathrm{~Hz}, 1 \mathrm{H}), 5.12(\mathrm{~d}, J=12 \mathrm{~Hz}, 1 \mathrm{H}), 5.15(\mathrm{~d}, J=12 \mathrm{~Hz}, 1 \mathrm{H}), 5.69-5.89(\mathrm{~m}, 1 \mathrm{H})$; ${ }^{13}$ C NMR (50 MHz): $\delta(\mathrm{ppm}) 13.6\left(\mathrm{CH}_{3}\right), 18.2\left(\mathrm{CH}_{2}\right), 36.1\left(\mathrm{CH}_{2}\right), 38.6\left(\mathrm{CH}_{3}\right), 39.0\left(\mathrm{CH}_{2}\right)$, $82.5(\mathrm{CH}), 118.8\left(\mathrm{CH}_{2}\right), 132.4(\mathrm{CH})$; EI-MS (RT): m/z (\%) 191 (1), 151 (72), 97 (20), 79 (59), 73 (20), 55 (100); HRMS: calcd. for $\mathrm{C}_{5} \mathrm{H}_{11} \mathrm{O}_{3} \mathrm{~S}$ [M- $\left.\mathrm{C}_{3} \mathrm{H}_{5}\right]^{+}$151.0428, found 151.0421 .

(S)-4-Azido-hept-1-ene (12). To a solution of 11 (4.50 g, $23.4 \mathrm{mmol})$ in DMSO (25 mL) was added portionwise $\mathrm{NaN}_{3}(3.04 \mathrm{~g}, 46.8 \mathrm{mmol})$ and the resulting suspension was stirred for $20 \mathrm{~h}$ at $50{ }^{\circ} \mathrm{C}$. After cooling the orange solution to r.t., $\mathrm{Et}_{2} \mathrm{O}(50 \mathrm{~mL})$ and $\mathrm{H}_{2} \mathrm{O}(50 \mathrm{~mL})$ were added and the aqueous layer was extracted with $\mathrm{Et}_{2} \mathrm{O}(3 \times 40 \mathrm{~mL})$. The combined organic layers were dried $\left(\mathrm{MgSO}_{4}\right)$ and the solvent was removed by distillation over a $10 \mathrm{~cm}$ vigreuxcolumn. 12 (3.15 g, 97\%) was afforded as yellowish oil, which was spectroscopically pure and subsequently used without further purification; $[\alpha]_{D}^{20}-21.4\left(c 0.87, \mathrm{CHCl}_{3}\right)$; IR (ATR): $v\left(\mathrm{~cm}^{-1}\right)$ 2961, 2936, 2875, 2100, 1643, 1259, 918; ${ }^{1} \mathbf{H}$ NMR $(200 \mathrm{MHz}): \delta(\mathrm{ppm}) 0.93(\mathrm{t}$, $J=7 \mathrm{~Hz}, 3 \mathrm{H}$ ), 1.34-1.55 (m, 4H), 2.26-2.33 (m, 2H), 3.33 (quin, $J=6 \mathrm{~Hz}, 1 \mathrm{H}), 5.15(\mathrm{~d}$, $J=13 \mathrm{~Hz}, 1 \mathrm{H}), 5.17(\mathrm{~d}, J=9 \mathrm{~Hz}, 1 \mathrm{H}), 5.71-5.92(\mathrm{~m}, 1 \mathrm{H}) ;{ }^{13} \mathbf{C} \mathbf{N M R}(50 \mathrm{MHz}): \delta(\mathrm{ppm}) 13.7$ $\left(\mathrm{CH}_{3}\right), 19.1\left(\mathrm{CH}_{2}\right), 35.9\left(\mathrm{CH}_{2}\right), 38.6\left(\mathrm{CH}_{2}\right), 61.9(\mathrm{CH}), 117.8\left(\mathrm{CH}_{2}\right), 133.9(\mathrm{CH})$; EI-MS (RT): m/z (\%) 111 (1), 70 (22), 57 (38), 55 (14), 43 (100), 41 (51); HRMS: calcd. for $\mathrm{C}_{7} \mathrm{H}_{13} \mathrm{~N}$ $\left[\mathrm{M}-\mathrm{N}_{2}\right]^{+} 111.1048$, found 111.1051 .

(S)-2-Nitro- $N$-(1-propyl-but-3-enyl)-benzenesulfonamide (6). To an ice-cold stirred suspension of $\mathrm{LiAlH}_{4}(1.79 \mathrm{~g}, 45.3 \mathrm{mmol})$ in dry $\mathrm{Et}_{2} \mathrm{O}(25 \mathrm{~mL})$ was added dropwise over $45 \mathrm{~min}$ a solution of $12(3.15 \mathrm{~g}, 22.6 \mathrm{mmol})$ in $\mathrm{Et}_{2} \mathrm{O}(25 \mathrm{~mL})$. The reaction mixture was allowed to warm to r.t. and stirred for $3 \mathrm{~h}$. After cooling the suspension to $0{ }^{\circ} \mathrm{C}$, aqueous $\mathrm{NaOH}(20 \mathrm{~mL}, 2.5 \mathrm{M})$ was added dropwise and stirred vigorously at r.t. for $45 \mathrm{~min}$. The solvent was decanted off and the remaining white solid was washed with $\mathrm{Et}_{2} \mathrm{O}$. After drying 
the combined ethereal layers $\left(\mathrm{MgSO}_{4}\right)$, the solvent was removed at atmospheric pressure by distillation over a $15 \mathrm{~cm}$ vigreux-column. The residue was solved in $\mathrm{CH}_{2} \mathrm{Cl}_{2}(15 \mathrm{~mL})$, before $\mathrm{K}_{2} \mathrm{CO}_{3}(6.25 \mathrm{~g}, 45.3 \mathrm{mmol})$ was added, and cooled to $0{ }^{\circ} \mathrm{C}$. After portionwise addition of 2-nitro-benzenesulfonyl chloride $(5.02 \mathrm{~g}, 22.6 \mathrm{mmol})$ under vigorously stirring, the resulting suspension was allowed to warm up to r.t. and stirred for $24 \mathrm{~h}$. The reaction mixture was diluted with $100 \mathrm{~mL} \mathrm{CH}_{2} \mathrm{Cl}_{2}$, washed with $\mathrm{H}_{2} \mathrm{O}(3 \times 30 \mathrm{~mL})$ and brine $(20 \mathrm{~mL})$, dried over $\mathrm{MgSO}_{4}$ and concentrated. The crude product was purified by flash chromatography (silica, MTBE/hexane 2:3) to give 6 as yellowish solid $(6.67 \mathrm{~g}, 89 \%)$ with an optical purity of 99\%ee; $\mathrm{R}_{\mathrm{f}}=0.38, \mathrm{mp}=35-37{ }^{\circ} \mathrm{C} ;[\alpha]_{D}^{20}-64.2\left(c 0.62, \mathrm{CHCl}_{3}\right) ; \mathbf{I R}(\mathrm{ATR}): v\left(\mathrm{~cm}^{-1}\right) 3345$, 2960, 2935, 2874, 1540, 1414, 1361, 1169; ${ }^{1} \mathbf{H}$ NMR (500 MHz): $\delta$ (ppm) 0.76 (t, $J=7 \mathrm{~Hz}$, $3 \mathrm{H}), 1.17-1.48(\mathrm{~m}, 4 \mathrm{H}), 2.09-2.19(\mathrm{~m}, 2 \mathrm{H}), 3.45-3.51(\mathrm{~m}, 1 \mathrm{H}), 4.85(\mathrm{~d}, J=13 \mathrm{~Hz}, 1 \mathrm{H}), 4.89$ $(\mathrm{d}, J=13 \mathrm{~Hz}, 1 \mathrm{H}), 5.23$ (d, $J=8 \mathrm{~Hz}, 1 \mathrm{H}), 5.49-5.57(\mathrm{~m}, 1 \mathrm{H}), 7.68-7.72(\mathrm{~m}, 2 \mathrm{H}), 7.79-7.81$ $(\mathrm{m}, 1 \mathrm{H}), 8.07-8.09(\mathrm{~m}, 1 \mathrm{H}) ;{ }^{13} \mathbf{C}$ NMR $(125 \mathrm{MHz}): \delta(\mathrm{ppm}) 13.7\left(\mathrm{CH}_{3}\right), 18.5\left(\mathrm{CH}_{2}\right), 36.9$ $\left(\mathrm{CH}_{2}\right), 39.6\left(\mathrm{CH}_{2}\right), 54.8(\mathrm{CH}), 118.6\left(\mathrm{CH}_{2}\right), 125.3(\mathrm{CH}), 130.5(\mathrm{CH}), 133.0(\mathrm{CH}), 133.2(\mathrm{CH})$, $133.6(\mathrm{CH}), 135.0\left(\mathrm{C}_{\mathrm{q}}\right), 147.7\left(\mathrm{C}_{\mathrm{q}}\right)$; EI-MS (RT): m/z (\%) 299 (1), 258 (16), 257 (84), 187 (16), 186 (100); HRMS: calcd. for $\mathrm{C}_{10} \mathrm{H}_{13} \mathrm{~N}_{2} \mathrm{O}_{4} \mathrm{~S}$ [M- $\left.\mathrm{C}_{3} \mathrm{H}_{5}\right]^{+}$257.0596, found 257.0591; Anal. calcd. for $\mathrm{C}_{13} \mathrm{H}_{18} \mathrm{~N}_{2} \mathrm{O}_{4} \mathrm{~S}$ : C 52.33, H 6.08, N 9.39, found C 52.47, H 6.11, N 9.24; Enantiomeric excess determined by HPLC, Chiracel OJ-column, flow rate: $0.3 \mathrm{ml} / \mathrm{min}$, temp. $20{ }^{\circ} \mathrm{C}$, eluent: hexane/propan-2-ol (6:4), retention times: $(S)$-enantiomer 23.93 min, $(R)$-enantiomer $26.04 \mathrm{~min}$.

(S)-2-Bromo-cyclohex-2-en-1-ol (15). Application of Me-CBS (16) as catalyst: To a solution of 16 (1 M in toluene, $4.00 \mathrm{mmol})$ in dry THF $(20 \mathrm{~mL})$ were added simultaneously solutions of $\mathbf{1 3}^{17}$ (4.67 $\left.\mathrm{g}, 26.7 \mathrm{mmol}\right)$ in $\mathrm{THF}(30 \mathrm{~mL})$ and $\mathrm{BH}_{3} \cdot \mathrm{SMe}_{2}\left(10 \mathrm{M}\right.$ in $\left.\mathrm{SMe}_{2}, 16.0 \mathrm{mmol}\right)$ in THF (20 mL) dropwise over $45 \mathrm{~min}$ at $-20{ }^{\circ} \mathrm{C}$. The mixture was stirred for further $45 \mathrm{~min}$ at $-20{ }^{\circ} \mathrm{C}$, then quenched with $\mathrm{MeOH}(30 \mathrm{~mL})$. After evaporation of the solvent, the residue was redissolved in $\mathrm{Et}_{2} \mathrm{O}$, washed with brine $(10 \mathrm{~mL})$, dried $\left(\mathrm{MgSO}_{4}\right)$ and concentrated. Chromatographic purification of the crude product (silica, $\mathrm{Et}_{2} \mathrm{O} /$ pentane 1:2) gave $\mathbf{1 5}$ as colourless oil $(4.67 \mathrm{~g}, 98 \%)$ and with an optical purity of $83 \% e e ; \mathrm{R}_{\mathrm{f}}=0.4$.

Application of MeO-CBS (17) as catalyst: Under Ar-atmosphere $(R)-\alpha, \alpha$-diphenylprolinol $(72.4 \mathrm{mg}, 0.29 \mathrm{mmol})$ and $\mathrm{B}(\mathrm{OMe})_{3}(38.9 \mu \mathrm{l}, 0.34 \mathrm{mmol})$ were dissolved in dry THF $(5 \mathrm{~mL})$. The mixture was stirred for $1 \mathrm{~h}$ at r.t., before $\mathrm{BH}_{3} \cdot N, N$-diethylaniline $(0.51 \mathrm{~mL}, 2.86 \mathrm{mmol})$ was added and the resulting solution cooled to $-10{ }^{\circ} \mathrm{C}$. A solution of $13^{17}(500 \mathrm{mg}$, $2.86 \mathrm{mmol})$ in dry THF (5 mL) was added over $45 \mathrm{~min}$. After complete conversion of the 
ketone (TLC-control), quenching was carried out by addition of $1 \mathrm{M} \mathrm{HCl}(5 \mathrm{~mL})$. The resulting mixture was diluted with $\mathrm{Et}_{2} \mathrm{O}$, washed with brine $(10 \mathrm{~mL})$, dried $\left(\mathrm{MgSO}_{4}\right)$ and concentrated. Chromatographic purification of the crude product (silica, $\mathrm{Et}_{2} \mathrm{O} /$ pentane 1:2) gave 15 as colourless oil (483 mg, 95\%) and with an enantiomeric excess of $99 \% ; R_{\mathrm{f}}=0.4$; $[\alpha]_{D}^{20}-85.2\left(c\right.$ 1.89, $\left.\mathrm{CHCl}_{3}\right)$; IR (ATR): $v\left(\mathrm{~cm}^{-1}\right)$ 3361, 2939, 2865, 2832, 1640, 1436, 1330, 1162, 1079, 1053, 992, 978, 936, 907, 832, 807, 748, 702; ${ }^{1} \mathbf{H}$ NMR (500 MHz): $\delta$ (ppm) 1.60-1.74 (m, 2H), 1.87-1.97 (m, 2H), 2.03-2.11 (m, 2H), 2.17 (d, J= 4 Hz, 1H), 4.19-4.21 $(\mathrm{m}, 1 \mathrm{H}), 6.20(\mathrm{t}, J=4 \mathrm{~Hz}, 1 \mathrm{H}) ;{ }^{13} \mathbf{C}$ NMR $(125 \mathrm{MHz}): \delta(\mathrm{ppm}) 17.6\left(\mathrm{CH}_{2}\right), 27.9\left(\mathrm{CH}_{2}\right), 32.5$ $\left(\mathrm{CH}_{2}\right), 69.7(\mathrm{CH}), 125.7\left(\mathrm{C}_{\mathrm{q}}\right), 132.5(\mathrm{CH})$; EI-MS (RT): m/z (\%) 176 (5), 148 (20), 97 (100), 79 (24), 55 (18); HRMS: calcd. for $\mathrm{C}_{6} \mathrm{H}_{9} \mathrm{BrO}[\mathrm{M}]^{+}$175.9837, found 175.9850; Enantiomeric excess was determined by HPLC, Chiracel OJ-column, flow rate: $1.0 \mathrm{ml} / \mathrm{min}$, temp.: $20{ }^{\circ} \mathrm{C}$, eluent: hexane/propan-2-ol (9:1), retention times: $(R)$-enantiomer $5.73 \mathrm{~min}$, $(S)$-enantiomer $6.58 \mathrm{~min}$.

(S)-Cyclohex-2-en-1-ol (5). To a solution of 15 (1.70 g, $9.60 \mathrm{mmol})$ in $\operatorname{dry~}_{2} \mathrm{O}(20 \mathrm{~mL})$ was added dropwise at $-78{ }^{\circ} \mathrm{C}$ tert-BuLi $(1.7 \mathrm{M}, 30.7 \mathrm{mmol})$ over $1 \mathrm{~h}$. After complete addition, the mixture was stirred for further $10 \mathrm{~min}$ at $-78{ }^{\circ} \mathrm{C}$ and was then warmed up to $-20{ }^{\circ} \mathrm{C}$ over $3 \mathrm{~h}$. The solution was quenched by addition of sat. $\mathrm{NaHCO}_{3}$-solution $(10 \mathrm{~mL})$ and stirred for $1 \mathrm{~h}$ at r.t. The layers were separated, the organic dried $\left(\mathrm{MgSO}_{4}\right)$ and evaporated. Chromatographic purification of the resulting crude product (silica, $\mathrm{Et}_{2} \mathrm{O} /$ pentane 1:2) afforded $5(895 \mathrm{mg}, 96 \%)$ as colourless oil; $\mathrm{R}_{\mathrm{f}}=0.32 ;[\alpha]_{D}^{20}-119.2\left(c 1.05, \mathrm{CHCl}_{3}\right)$; IR (ATR): $v\left(\mathrm{~cm}^{-1}\right)$ 3328, 3024, 2934, 2861, 2837, 1650, 1450, 1282, 1049, 959, 897, 726, 670; ${ }^{1}$ H NMR (500 MHz): $\delta$ (ppm) 1.48-1.53 (m, 2H), 1.65-1.78 (m, 2H), 1.88-1.95 (m, 2H), 2.76$2.78(\mathrm{~m}, 1 \mathrm{H}), 4.10(\mathrm{bs}, 1 \mathrm{H}), 5.64-5.67(\mathrm{~m}, 1 \mathrm{H}), 5.72-5.87(\mathrm{~m}, 1 \mathrm{H}) ;{ }^{13} \mathbf{C} \mathbf{N M R}(125 \mathrm{MHz})$ : $\delta(\mathrm{ppm})=19.1\left(\mathrm{CH}_{2}\right), 25.0\left(\mathrm{CH}_{2}\right), 31.9\left(\mathrm{CH}_{2}\right), 65.4(\mathrm{CH}), 130.1(\mathrm{CH}), 130.3(\mathrm{CH}) ; \mathbf{E I}-\mathbf{M S}$ (RT): m/z (\%) 98 (33), 97 (42), 83 (49), 79 (23), 70 (100), 69 (27), 55 (31), 41 (28); HRMS: calcd. for $\mathrm{C}_{6} \mathrm{H}_{10} \mathrm{O}[\mathrm{M}]^{+}$98.0731, found 98.0733.

\section{$N$-(R)-Cyclohex-2-enyl-2-nitro- $N$-((S)-1-propyl-but-3-enyl)-benzenesulfonamide}

$6(2.00 \mathrm{~g}, 6.70 \mathrm{mmol})$, triphenylphosphine (3.52 g, $13.4 \mathrm{mmol})$ and 5 (1.30 g, $13.4 \mathrm{mmol})$ were dissolved in dry THF (30 ml) and cooled to $0{ }^{\circ} \mathrm{C}$. DIAD (2.66 ml, $\left.13.4 \mathrm{mmol}\right)$ was added dropwise over $30 \mathrm{~min}$ to the mixture and stirred for $72 \mathrm{~h}$ at r.t. After evaporation of the solvent, the crude product was purified by chromatography (silica, MTBE/hexane 1:2) to give 4 as yellowish oil $(2.10 \mathrm{~g}, 83 \%) ; \mathrm{R}_{\mathrm{f}}=0.36 ;[\alpha]_{D}^{20}-25.7\left(c 0.99, \mathrm{CHCl}_{3}\right) ; \mathbf{I R}(\mathrm{ATR}): v\left(\mathrm{~cm}^{-1}\right)$ 
2958, 2934, 2872, 1545, 1373, 1346, 1163; ${ }^{1} \mathbf{H}$ NMR $(200 \mathrm{MHz}): \delta(\mathrm{ppm}) 0.76(\mathrm{t}, J=7 \mathrm{~Hz}$, 3H), 1.11-1.29 (m, 2H), 1.57-1.73 (m, 4H), 1.83-2.00 (m, 4H), 2.49-2.56 (m, 2H), 3.48 (bs, $1 \mathrm{H}), 4.39$ (bs, 1H), 4.95 (d, $J=11 \mathrm{~Hz}, 1 \mathrm{H}), 5.05$ (d, $J=11 \mathrm{~Hz}, 1 \mathrm{H}), 5.55$ (d, $J=11 \mathrm{~Hz}, 1 \mathrm{H})$, 5.61-5.74 (m, 1H), 5.79-5.87 (m, 1H), 7.51-7.55 (m, 1H), 7.63-7.67 (m, 2H), 8.04-8.08 (m, $1 \mathrm{H}) ;{ }^{13} \mathrm{C}$ NMR $(50 \mathrm{MHz}): \delta(\mathrm{ppm}) 13.7\left(\mathrm{CH}_{3}\right), 20.3\left(\mathrm{CH}_{2}\right), 22.2\left(\mathrm{CH}_{2}\right), 24.3\left(\mathrm{CH}_{2}\right), 29.6$ $\left(\mathrm{CH}_{2}\right), 35.6\left(\mathrm{CH}_{2}\right), 40.8\left(\mathrm{CH}_{2}\right), 50.8(\mathrm{CH}), 53.3(\mathrm{CH}), 56.2(\mathrm{CH}), 58.7(\mathrm{CH}), 116.9\left(\mathrm{CH}_{2}\right)$, $123.5(\mathrm{CH}), 128.7(\mathrm{CH}), 130.6(\mathrm{CH}), 131.0(\mathrm{CH}), 132.9(\mathrm{CH}), 135.0\left(\mathrm{C}_{\mathrm{q}},\right), 135.6\left(\mathrm{C}_{\mathrm{q}}\right)$; EI-MS $\left(130{ }^{\circ} \mathrm{C}\right): \mathrm{m} / \mathrm{z}(\%) 378$ (1), 337 (12), 257 (100), 186 (54), 81 (80); HRMS: calcd. for $\mathrm{C}_{16} \mathrm{H}_{21} \mathrm{~N}_{2} \mathrm{O}_{4} \mathrm{~S}\left[\mathrm{M}-\mathrm{C}_{3} \mathrm{H}_{5}\right]^{+}$337.1222, found 337.1220; Anal. calcd. for $\mathrm{C}_{19} \mathrm{H}_{26} \mathrm{~N}_{2} \mathrm{O}_{4} \mathrm{~S}$ : C 60.30, H 6.92, N 7.40, found C 60.16, H 6.91, N 7.41.

\section{(2S,6R)- $N$-(2-Nitrobenzenesulfonyl)-6-pent-4-enyl-2-propyl-1,2,3,6-tetrahydropyridine}

(3a). A solution of $4(1.00 \mathrm{~g}, 2.64 \mathrm{mmol})$ in $\mathrm{CH}_{2} \mathrm{Cl}_{2}(80 \mathrm{~mL})$ was saturated with ethylene via a syringe, before Grubbs I catalyst (109 mg, $5 \mathrm{~mol} \%$ ) was added portionwise. The reaction mixture was stirred for $24 \mathrm{~h}$ at $50{ }^{\circ} \mathrm{C}$ under ethylene-atmosphere in a sealed vial. After cooling the resulting brown solution to r.t. and removing the solvent in vacuo, the crude product was purified by flash-chromatography (silica, MTBE/hexane 1:3) to afford 3a $(963 \mathrm{mg}, 96 \%)$ as yellowish oil and with an optical purity of $98 \% e e ; \mathrm{R}_{\mathrm{f}}=0.26 ;[\alpha]_{D}^{20}-163.8$ (c 0.61, $\mathrm{CHCl}_{3}$ ); IR (ATR): $v\left(\mathrm{~cm}^{-1}\right)$ 2957, 2933, 2872, 1544, 1373, 1348, 1174; ${ }^{\mathbf{1}} \mathbf{H}$ NMR (500 MHz): $\delta(\mathrm{ppm}) 0.93(\mathrm{t}, J=7 \mathrm{~Hz}, 3 \mathrm{H}), 1.34-1.82(\mathrm{~m}, 8 \mathrm{H}), 1.85-1.96(\mathrm{~m}, 2 \mathrm{H}), 2.05-2.15$ (m, 2H), 3.99-4.03 (m, 1H), 4.27 (bs, 1H), 4.98 (dd, $J=3,13 \mathrm{~Hz}, 1 \mathrm{H}), 5.03$ (dd, $J=3,10$ $\mathrm{Hz}, 1 \mathrm{H}), 5.60-5.68(\mathrm{~m}, 1 \mathrm{H}), 5.71-5.79(\mathrm{~m}, 1 \mathrm{H}), 5.82-5.91(\mathrm{~m}, 1 \mathrm{H}), 7.54-7.58(\mathrm{~m}, 1 \mathrm{H}), 7.61-$ $7.67(\mathrm{~m}, 2 \mathrm{H}), 7.91-7.95(\mathrm{~m}, 1 \mathrm{H}) ;{ }^{13} \mathbf{C}$ NMR (125 MHz): $\delta(\mathrm{ppm}) 13.8\left(\mathrm{CH}_{3}\right), 20.1\left(\mathrm{CH}_{2}\right), 26.2$ $\left(\mathrm{CH}_{2}\right), 27.1\left(\mathrm{CH}_{2}\right), 33.6\left(\mathrm{CH}_{2}\right), 37.2\left(\mathrm{CH}_{2}\right), 37.3\left(\mathrm{CH}_{2}\right), 50.9(\mathrm{CH}), 54.0(\mathrm{CH}), 114.9\left(\mathrm{CH}_{2}\right)$, $122.9(\mathrm{CH}), 124.1(\mathrm{CH}), 125.9(\mathrm{CH}), 130.4(\mathrm{CH}), 131.5(\mathrm{CH}), 133.2(\mathrm{CH}), 134.2\left(\mathrm{C}_{\mathrm{q}}\right), 138.4$ (CH), $149.2\left(\mathrm{C}_{\mathrm{q}}\right)$; EI-MS (100 $\left.{ }^{\circ} \mathrm{C}\right): \mathrm{m} / \mathrm{z}$ (\%) 378 (1), 310 (20), 309 (100), 257 (16), 186 (45); HRMS: calcd. for $\mathrm{C}_{19} \mathrm{H}_{26} \mathrm{~N}_{2} \mathrm{O}_{4} \mathrm{~S}[\mathrm{M}]^{+}$378.1613, found 378.1622; Anal. calcd. for $\mathrm{C}_{19} \mathrm{H}_{26} \mathrm{~N}_{2} \mathrm{O}_{4} \mathrm{~S}$ : C 60.30, H 6.92, N 7.40, found C 60.09, H 6.91, N 7.39; Enantiomeric excess was determined by HPLC, Chiracel OJ-column, flow rate: $0.7 \mathrm{ml} / \mathrm{min}$, temp.: $20{ }^{\circ} \mathrm{C}$, eluent: hexane/propan-2-ol (91:9); retention times: (2R,6S)-enantiomer $17.15 \mathrm{~min},(2 S, 6 R)$ enantiomer $18.01 \mathrm{~min}$. 
(2S,6R)-N-Benzyl-6-pent-4-enyl-2-propyl-1,2,3,6-tetrahydropyridine (3b). To a stirred solution of $\mathrm{K}_{2} \mathrm{CO}_{3}(876 \mathrm{mg}, 6.34 \mathrm{mmol})$ and $3 \mathbf{a}(300 \mathrm{mg}, 0.79 \mathrm{mmol})$ in DMF (7 mL), was added dropwise thiophenol $(89.1 \mu \mathrm{l}, 0.87 \mathrm{mmol})$ at $80{ }^{\circ} \mathrm{C}$. The mixture was stirred for $2 \mathrm{~h}$, before TBAI (14.6 mg, $0.04 \mathrm{mmol})$ and benzyl bromide $(0.47 \mathrm{~mL}, 3.96 \mathrm{mmol})$ were added. The resulting bright yellow solution was stirred for $1 \mathrm{~h}$, cooled to r.t. diluted with MTBE $(50 \mathrm{~mL})$ and $\mathrm{H}_{2} \mathrm{O}(50 \mathrm{~mL})$ added. The aqueous layer was extracted with MTBE $(2 \times 30 \mathrm{~mL})$ and the combined organic layers were washed with brine $(15 \mathrm{~mL})$ and concentrated. The residue was redissolved in hexane $(40 \mathrm{~mL})$ and extracted with $1 \mathrm{M} \mathrm{HCl}(3 \times 30 \mathrm{~mL})$. To the combined aqueous layers was added MTBE $(40 \mathrm{~mL})$ and the mixture was saturated with $\mathrm{K}_{2} \mathrm{CO}_{3}$ under vigorous stirring. After separation of the layers, the aqueous layer was extracted with MTBE $(2 \times 30 \mathrm{~mL})$ and the combined organic layers were dried $\left(\mathrm{MgSO}_{4}\right)$. Evaporation of the solvent and chromatographic purification of the crude product (alumina, hexane $/ \mathrm{CH}_{2} \mathrm{Cl}_{2}$ 10:1) gave $3 \mathbf{b}(213 \mathrm{mg}, 95 \%)$ as colourless oil; $[\alpha]_{D}^{20}-36.7\left(c\right.$ 1.63, $\left.\mathrm{CHCl}_{3}\right)$; IR (ATR): $v\left(\mathrm{~cm}^{-1}\right)$ 3075, 3024, 2955, 2929, 2870,1640, 1494, 1435, 909, 728, 697; ${ }^{1} \mathbf{H}$ NMR (200 MHz): $\delta(\mathrm{ppm}) 0.82(\mathrm{t}, J=7 \mathrm{~Hz}, 3 \mathrm{H}), 1.27-1.62(\mathrm{~m}, 8 \mathrm{H}), 1.86-1.95(\mathrm{~m}, 2 \mathrm{H}), 2.17-2.20$ (m, 2H), 2.76-2.84 (m, 1H), 3.05 (bs, 1H), 3.72 (s, 2H), 4.87 (dd, J = 2, 17 Hz, 1H), 4.93 (dd, $J=2,13 \mathrm{~Hz}, 1 \mathrm{H}), 5.62-5.65(\mathrm{~m}, 1 \mathrm{H}), 5.66-5.71(\mathrm{~m}, 1 \mathrm{H}), 5.73-5.82(\mathrm{~m}, 1 \mathrm{H}), 7.19-7.38$ (m, $5 \mathrm{H}) ;{ }^{13} \mathrm{C}$ NMR $(50 \mathrm{MHz}): \delta(\mathrm{ppm}) 14.1\left(\mathrm{CH}_{3}\right), 19.9\left(\mathrm{CH}_{2}\right), 25.9\left(\mathrm{CH}_{2}\right), 26.0\left(\mathrm{CH}_{2}\right), 33.7$ $\left(\mathrm{CH}_{2}\right), 34.6\left(\mathrm{CH}_{2}\right), 36.8\left(\mathrm{CH}_{2}\right), 56.0\left(\mathrm{CH}_{2}\right), 56.7(\mathrm{CH}), 59.4(\mathrm{CH}), 114.0\left(\mathrm{CH}_{2}\right), 123.7(\mathrm{CH})$, $126.2(\mathrm{CH}), 127.8(\mathrm{CH}), 128.2(\mathrm{CH}), 129.3(\mathrm{CH}), 138.9(\mathrm{CH}), 141.8\left(\mathrm{C}_{\mathrm{q}}\right)$; EI-MS $(\mathrm{RT}): \mathrm{m} / \mathrm{z}$ (\%) 283 (4), 240 (30), 215 (22), 214 (100), 91 (94); HRMS: calcd. for $\mathrm{C}_{20} \mathrm{H}_{29} \mathrm{~N}[\mathrm{M}]^{+}$ 283.2300, found 283.2303; Anal. calcd. for $\mathrm{C}_{20} \mathrm{H}_{29} \mathrm{~N}$ : C 84.75, H 10.31, N 4.94, found C 84.35, H 9.93, N 5.02.

\section{(2S,4aR,5S,8aR)- $N$-Benzyl-5-methyl-2-propyl-decahydroquinoline $\quad(18) . \quad \mathrm{Cp}_{2} \mathrm{ZrCl}_{2}$}

(309 mg, $1.06 \mathrm{mmol}$ ) was dissolved under argon-atmosphere in dry THF (30 mL) and cooled to $-78{ }^{\circ} \mathrm{C}$, before $n$-BuLi $(1.6 \mathrm{M}, 2.12 \mathrm{mmol})$ was added dropwise and stirred for $45 \mathrm{~min}$. To the reaction mixture was added dropwise a solution of $\mathbf{3 b}(300 \mathrm{mg}, 1.06 \mathrm{mmol})$ in dry THF $(10 \mathrm{~mL})$ and stirred for further $45 \mathrm{~min}$. The yellow solution was allowed to warm up to r.t. and was stirred for $5 \mathrm{~h}$. The resulting orange solution was quenched by dropwise addition of $1 \mathrm{M} \mathrm{HCl}(10 \mathrm{~mL})$, stirred for $30 \mathrm{~min}$ and saturated with $\mathrm{K}_{2} \mathrm{CO}_{3}$. After separation of the layers, the aqueous layer was extracted with $\operatorname{MTBE}(2 \times 30 \mathrm{~mL})$ and the combined organic layers were dried $\left(\mathrm{MgSO}_{4}\right)$ and concentrated. The resulting crude product was purified by chromatography on alumina $\left(\mathrm{CH}_{2} \mathrm{Cl}_{2} /\right.$ hexane 1:20) to afford $18(224 \mathrm{mg}, 74 \%)$ as colourless 
solid; mp. $=29-31{ }^{\circ} \mathrm{C} ;[\alpha]_{D}^{20}+15.5\left(c 0.75, \mathrm{CHCl}_{3}\right)$; IR (ATR): $v\left(\mathrm{~cm}^{-1}\right) 2925,2858,1603$, 1493, 1452, 1375, 727, 696; ${ }^{1} \mathbf{H}$ NMR (200 MHz): $\delta(\mathrm{ppm}) 0.77$ (t, $\left.J=7 \mathrm{~Hz}, 3 \mathrm{H}\right), 0.88(\mathrm{~d}$, $J=6 \mathrm{~Hz}, 3 \mathrm{H}), 0.93-1.07(\mathrm{~m}, 4 \mathrm{H}), 1.13-1.43(\mathrm{~m}, 6 \mathrm{H}), 1.45-1.53(\mathrm{~m}, 1 \mathrm{H}), 1.58-1.64(\mathrm{~m}, 2 \mathrm{H})$, 1.67-1.70 (m, 1H), 1.76-1.78 (m, 1H), 1.98-2.02 (m, 1H), 2.19-2.30 (m, 1H), 2.45-2.55 (m, 1H), $3.64(\mathrm{~d}, J=17 \mathrm{~Hz}, 1 \mathrm{H}), 3.75(\mathrm{~d}, J=17 \mathrm{~Hz}, 1 \mathrm{H}), 7.16(\mathrm{t}, J=7 \mathrm{~Hz}, 1 \mathrm{H}), 7.27$ (t, $J=7 \mathrm{~Hz}$, 2H), $7.38(\mathrm{~d}, J=7 \mathrm{~Hz}, 2 \mathrm{H}) ;{ }^{13} \mathbf{C}$ NMR (50 MHz): $\delta(\mathrm{ppm}) 14.2\left(\mathrm{CH}_{3}\right), 19.4\left(\mathrm{CH}_{3}\right), 19.6$ $\left(\mathrm{CH}_{2}\right), 25.2\left(\mathrm{CH}_{2}\right), 28.5\left(\mathrm{CH}_{2}\right), 29.3\left(\mathrm{CH}_{2}\right), 32.7\left(\mathrm{CH}_{2}\right), 35.4\left(\mathrm{CH}_{2}\right), 37.1(\mathrm{CH}), 37.5\left(\mathrm{CH}_{2}\right)$, $43.8(\mathrm{CH}), 51.1\left(\mathrm{CH}_{2}\right), 63.0(\mathrm{CH}), 68.1(\mathrm{CH}), 125.6(\mathrm{CH}), 127.2(\mathrm{CH}), 127.7(\mathrm{CH}), 143.9$ $\left(\mathrm{C}_{\mathrm{q}}\right)$; EI-MS (RT): m/z (\%) 285 (1), 241 (26), 242 (100), 91 (48); HRMS: calcd. for $\mathrm{C}_{20} \mathrm{H}_{31} \mathrm{~N}$ $[\mathrm{M}]^{+}$285.2456, found 285.2455; Anal. calcd. for $\mathrm{C}_{20} \mathrm{H}_{31} \mathrm{~N}$ : C 84.15, H 10.95, N 4.91, found C 84.15, H 10.86, N 4.91.

(2S,4aR,5S,8aR)-5-Methyl-2-propyl-decahydroquinoline (1). To a suspension of palladium on charcoal $(10 \% \mathrm{Pd} / \mathrm{C}, 32.0 \mathrm{mg})$ in $\mathrm{MeOH}(6 \mathrm{~mL})$ was added a solution of 18 (170 mg, $0.59 \mathrm{mmol})$ in $\mathrm{MeOH}(6 \mathrm{~mL})$. The reaction mixture was flushed with $\mathrm{H}_{2}$ and stirred under balloon pressure over night. After filtration of the suspension, the resulting solution was dried $\left(\mathrm{MgSO}_{4}\right)$ and concentrated. The crude product was purified by bulb to bulb distillation (0.05 mbar, $\left.150{ }^{\circ} \mathrm{C}\right)$ to give $\mathbf{1}(105 \mathrm{mg}, 90 \%)$ as colourless solid; $\mathrm{mp} .=29-30{ }^{\circ} \mathrm{C}$; $[\alpha]_{D}^{20}+27.4\left(c\right.$ 0.56, MeOH); IR (ATR): $v\left(\mathrm{~cm}^{-1}\right)$ 3348, 2959, 2922, 2868, 2736, 2681, 2536, 1587, 1449, 1041; ${ }^{1} \mathbf{H}$ NMR (500 MHz): $\delta$ (ppm) 0.64-0.71 (m, 1H), 0.83 (d, J= $\left.7 \mathrm{~Hz}, 3 \mathrm{H}\right)$, 0.85-0.86 (m, 1H), 0.88 (t, $J=4 \mathrm{~Hz}, 3 \mathrm{H}), 0.93-1.10(\mathrm{~m}, 3 \mathrm{H}), 1.19-1.38(\mathrm{~m}, 6 \mathrm{H}), 1.58-1.63(\mathrm{~m}$, 2H), 1.66-1.70 (m, 2H), 1.93-1.96 (m, 2H), 2.12-2.17 (m, 1H), 2.46-2.49 (m, 1H); ${ }^{13}$ C NMR (125 MHz): $\delta(\mathrm{ppm}) 14.3\left(\mathrm{CH}_{3}\right), 19.2\left(\mathrm{CH}_{3}\right), 24.7\left(\mathrm{CH}_{2}\right), 29.1\left(\mathrm{CH}_{2}\right), 32.9\left(\mathrm{CH}_{2}\right), 33.1\left(\mathrm{CH}_{2}\right)$, $34.0\left(\mathrm{CH}_{2}\right), 35.7\left(\mathrm{CH}_{2}\right), 36.3(\mathrm{CH}), 39.5\left(\mathrm{CH}_{2}\right), 48.6(\mathrm{CH}), 56.6(\mathrm{CH}), 61.4(\mathrm{CH})$; EI-MS $\left(100{ }^{\circ} \mathrm{C}\right): \mathrm{m} / \mathrm{z}(\%) 196$ (18), 195 (72), 194 (80), 152 (100); HRMS: calcd. for $\mathrm{C}_{13} \mathrm{H}_{25} \mathrm{~N}$ 195.1987, found 195.1988; Anal. calcd. for $\mathrm{C}_{13} \mathrm{H}_{25} \mathrm{~N}$ : C 79.93, H 12.90, N 7.17, found C 79.98, H 12.92, N 7.08. 